\title{
OPTIMAL DIVIDENDS IN THE DUAL MODEL WITH DIFFUSION
}

BY

\author{
Benjamin Avanzi and Hans U. Gerber
}

\begin{abstract}
In the dual model, the surplus of a company is a Lévy process with sample paths that are skip-free downwards. In this paper, the aggregate gains process is the sum of a shifted compound Poisson process and an independent Wiener process. By means of Laplace transforms, it is shown how the expectation of the discounted dividends until ruin can be calculated, if a barrier strategy is applied, and how the optimal dividend barrier can be determined. Conditions for optimality are discussed and several numerical illustrations are given. Furthermore, a family of models is analysed where the individual gain amount distribution is rescaled and compensated by a change of the Poisson parameter.
\end{abstract}

\section{KEYWORDS}

Optimal dividends, Barrier strategies, Dual model, Smooth pasting condition, Jump-diffusion, Laplace transforms.

\section{INTRODUCTION}

How much of the surplus should a stock company distribute to its shareholders? A possible goal is to maximize the expectation of the discounted dividends before ruin of the company; a survey of the related literature can be found in Avanzi (2008). The question was first addressed by de Finetti (1957). In a model where the periodic gains of a company take on only the values -1 and +1 , he showed that the optimal dividend strategy is a barrier strategy. In the classical model of risk theory, the surplus of a company at time $t$ (before dividends are introduced) is

$$
u+c t-S(t), \quad t \geq 0
$$

Here $u$ is the initial surplus, $c$ is the constant rate at which premiums are received, and $S(t)$ denotes the aggregate claims by time $t$. In this model, the optimal dividend strategy is not a barrier strategy in general, and finding it is a challenge; see, for example, Bühlmann (1970), Azcue and Muler (2005) and Schmidli (2008). 
Avanzi et al (2007) consider the dual model. Now the surplus of the company at time $t$ (before the introduction of dividends) is

$$
u-c t+S(t), \quad t \geq 0
$$

Here $u$ is again the initial surplus, but $c$ is the constant rate at which expenses occur and $S(t)$ represents the aggregate gains.

Both (1.1) and (1.2) define collective models. The classical model (1.1) is appropriate when the occurence of certain events results in a loss and the nonoccurence results in a gain. The dual model (1.2) seems appropriate when the occurence of certain events results in a gain and the non-occurence results in a loss. This is the case for a portfolio of life annuities, where the risk consists of survival and the event death leads to gains. Furthermore, the dual model seems appropriate for a company that specializes in inventions and discoveries.

In this paper we examine the dual model that is perturbed by diffusion. The surplus at time $t$ is now

$$
u-c t+S(t)+\sigma W(t), \quad t \geq 0
$$

where $\{S(t)\}$ is a compound Poisson process, with Poisson parameter $\lambda$ and probability density function $p(y), y \geq 0$, of the individual gains and $\{W(t)\}$ is a standard Wiener process that is independent of $\{S(t)\}$. The diffusion term adds uncertainty to the expenses an brings the model a step closer to reality. We shall assume that

$$
E[S(1)]-c>0
$$

that is, that the expected gain per unit time is positive. In the following, it will be denoted by the symbol $\mu$.

In the dual model, the optimal dividend strategy is a barrier strategy. This follows essentially from a result found by Miyasawa (1962). He generalized the de Finetti model and assumed that the periodic gains of a company have the values $-1,0,+1,+2,+3, \ldots$. For this model he showed that the optimal dividend strategy is a barrier strategy. The continuous counterpart of this is a model where the surplus (before dividends) is a Lévy process with sample paths that are skip-free downwards. From Miyasawa's result it follows by analogy that the optimal dividend strategy in the dual model is also a barrier strategy. A direct proof that the optimal strategy is a barrier strategy is of some interest but has not been given to our knowledge; the proof in Bayraktar and Egami (2008) is for exponential gains only.

The paper is organized as follows. In Section 2, for a given barrier $b$, we consider $V(u ; b)$, the expected discounted value of the dividends until ruin, a function of the initial surplus $u$. It is shown that $V(u ; b)$ can be characterized as the solution of a second order integro-differential equation in conjunction with two boundary conditions. In Section 3, we consider the special case where 
the gains distribution is a mixture or a combination of exponential distributions. Then the integro-differential equation leads to a differential equation, and $V(u ; b)$ can be obtained in a transparent way. Section 4 explains the more general method of Laplace transforms. This method is somewhat indirect: given the value of $V(b ; b)$, the underlying value of $b$ is determined as a zero of a certain function. In Section 5, the optimal dividend barrier $b^{*}$ is introduced. It is shown that $V\left(b^{*} ; b^{*}\right)=\mu / \delta$. This result is crucial for the determination of $b^{*}$ and $V\left(u^{*} ; b^{*}\right)$ by the method of Laplace transforms. Furthermore, $b^{*}$ can be obtained as a zero of a certain determinant. In Section 6, a family of models is considered. The distribution of an individual gain is rescaled and the Poisson parameter is adjusted such that the expected gain per unit time is unchanged.

\section{THE VALUE OF A BARRIER STRATEGY}

Suppose that the dividends are paid according to a barrier strategy, say with parameter $b$. Whenever the surplus exceeds (or is about to exceed) the level $b$, the excess is paid out immediately as a dividend. Let $D(t)$ denote the aggregate dividends by time $t$. Then

$$
D(t)=\max _{0 \leq \tau \leq t}(u-c \tau+S(\tau)+\sigma W(\tau)-b)_{+},
$$

and the (modified) surplus at time $t$ is

$$
U(t)=u-c t+S(t)+\sigma W(t)-D(t) .
$$

Note that there are two kinds of dividends, single dividend payments when the surplus jumps beyond $b$, and a series of "small" payments due to the oscillating nature of the sample path when the surplus is at $b$. This is illustrated in Figure 1.

Let $V(u ; b)=E\left[\int_{0}^{T} e^{-\delta t} \mathrm{~d} D(t)\right]$ denote the expectation of the discounted dividends until the time of ruin $T$ (when $U(t)=0$ for the first time), where $\delta>0$ is the force of interest to discount the dividends. Note that

$$
V(u ; b)=u-b+V(b ; b), u>b .
$$

For $0 \leq u \leq b$, the function $V(u ; b)$ is determined by the integro-differential equation

$$
\begin{aligned}
& \frac{1}{2} \sigma^{2} V^{\prime \prime}(u ; b)-c V^{\prime}(u ; b)-(\lambda+\delta) V(u ; b)+\lambda \int_{0}^{b-u} V(u+y ; b) p(y) \mathrm{d} y \\
& +\lambda \int_{b-u}^{\infty}(u+y-b) p(y) \mathrm{d} y+\lambda V(b ; b)[1-P(b-u)]=0,
\end{aligned}
$$




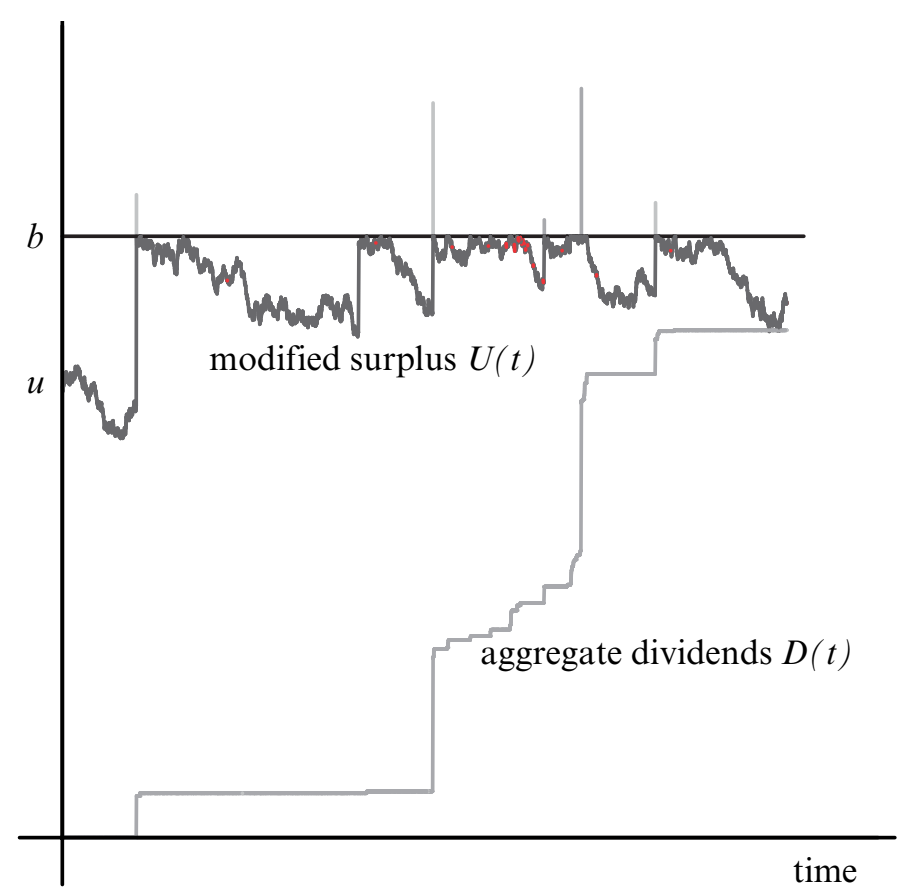

FIGURE 1: Typical sample paths of $U(t)$ and $D(t)$.

in combination with the boundary conditions

$$
V(0 ; b)=0
$$

and

$$
V^{\prime}(b-; b)=1 .
$$

Equation (2.4) can be obtained by the following heuristic reasoning. Let $0<$ $u<b$ and consider an infinitesimal time interval of length $\mathrm{d} t$. Then by conditioning, we see that

$$
\begin{aligned}
& V(u ; b)= \\
& e^{-(\lambda+\delta) \mathrm{d} t} E[V(u-c \mathrm{~d} t+\sigma W(\mathrm{~d} t) ; b)]+\lambda \mathrm{d} t \int_{0}^{\infty} V(u+y ; b) p(y) \mathrm{d} y .
\end{aligned}
$$

Noting that

$$
E[V(u-c \mathrm{~d} t+\sigma W(\mathrm{~d} t) ; b)]=V(u ; b)-c V^{\prime}(u ; b) \mathrm{d} t+\frac{1}{2} \sigma^{2} V^{\prime \prime}(u ; b) \mathrm{d} t,
$$


we expand $e^{-(\lambda+\delta) \mathrm{d} t}$, substract $V(u ; b)$ on either side of (2.7), divide by $\mathrm{d} t$ and let $\mathrm{d} t \rightarrow 0$. This yields the equation

$\frac{1}{2} \sigma^{2} V^{\prime \prime}(u ; b)-c V^{\prime}(u ; b)-(\lambda+\delta) V(u ; b)+\lambda \int_{0}^{\infty} V(u+y ; b) p(y) \mathrm{d} y=0$.

From this and (2.3) we obtain the integro-differential equation (2.4).

Condition (2.5) is obvious: if $u=0$, ruin is immediate, and no dividends can be paid. To understand condition (2.6), compare two situations, $u=b$ and $u=$ $b-\mathrm{d} u$. In the second case, the surplus will reach the barrier after an instant (because of oscillation) and from thereon, the two sample paths will be the same. At this time, a dividend of $\mathrm{d} u$ will have been paid in the first case. It follows that

$$
V(b ; b)=V(b-\mathrm{d} u ; b)+\mathrm{d} u,
$$

which explains (2.6).

Remark 2.1. From (2.4) with $u=b$ and (2.6) we obtain the result that

$$
V(b ; b)=\frac{\mu+\frac{1}{2} \sigma^{2} V^{\prime \prime}(b-; b)}{\delta},
$$

where $\mu$ is given by (1.4).

Remark 2.2. Model (1.2) is in a sense the limit of model (1.3) if $\sigma \rightarrow 0$. For example, (2.4) with $\sigma=0$ is (2.3) in Avanzi et al (2007). But not any formula for model (1.3) yields a valid formula in model (1.2), if we set $\sigma=0$. For example, formula (2.6) is not valid in model (1.2). And, if we set $\sigma=0$ in (2.11), we seem to get $V(b ; b)=\mu / \delta$, which is not true for arbitrary $b$.

\section{Mixtures of EXPONENTIAL GAins Distributions}

Suppose that

$$
p(y)=\sum_{i=1}^{n} A_{i} \beta_{i} e^{-\beta_{i} y}, \quad y>0
$$

where $\beta_{1}<\beta_{2}<\ldots<\beta_{n}, A_{i}>0$, and $A_{1}+\ldots+A_{n}=1$. We show how $V(u ; b)$ can be computed, and some light will be shed on the limit $\sigma \rightarrow 0$, that is, how $V(u ; b)$ is obtained as a limit when model (1.3) is replaced by model (1.2).

To obtain $V(u ; b)$, we generalize the calculations in Avanzi et al (2007, Section 4), which is why details are omitted. In (2.4), we replace the integration variable $y$ by $x=u+y$ and apply the operator 


$$
\left(\frac{\mathrm{d}}{\mathrm{d} u}-\beta_{1}\right)\left(\frac{\mathrm{d}}{\mathrm{d} u}-\beta_{2}\right) \cdots\left(\frac{\mathrm{d}}{\mathrm{d} u}-\beta_{n}\right)
$$

to the resulting equation, to see that $V(u ; b)$ satisfies a linear homogeneous differential equation (with constant coefficients) of order $n+2$. Thus

$$
V(u ; b)=\sum_{k=0}^{n+1} C_{k} e^{r_{k} u}, \quad 0 \leq u \leq b .
$$

Upon substitution in (2.4) we see that $r_{0}, r_{1}, \ldots, r_{n+1}$ are the solutions of the equation

$$
\frac{1}{2} \sigma^{2} \xi^{2}-c \xi-(\lambda+\delta)+\lambda \sum_{i=1}^{n} A_{i} \frac{\beta_{i}}{\beta_{i}-\xi}=0,
$$

which is equivalent to a polynomial equation of degree $n+2$. One can show that

$$
r_{0}<0<r_{1}<\beta_{1}<\ldots<r_{n}<\beta_{n}<r_{n+1}
$$

Furthermore, the coefficients $C_{k}=C_{k}(b)$ satisfy the equations

$$
\sum_{k=0}^{n+1} C_{k} \frac{r_{k}}{\beta_{i}-r_{k}} e^{r_{k} b}=\frac{1}{\beta_{i}}, \quad i=1, \ldots, n .
$$

Finally, from conditions (2.5) and (2.6) we gather that

$$
\begin{aligned}
& \sum_{k=0}^{n+1} C_{k}=0, \\
& \sum_{k=0}^{n+1} C_{k} r_{k} e^{r_{k} b}=1 .
\end{aligned}
$$

Equations (3.5)-(3.7) constitute a system of $n+2$ linear equations to determine $C_{0}, C_{1}, \ldots, C_{n+1}$.

Illustration 3.1. For Table 1, it is assumed that $b=10, p(y)=e^{-y}, \lambda=1, c=0.75$ and $\delta=0.005$. The coefficients $r_{0}, r_{1}, r_{2}$ are the solutions of (3.3), and $C_{0}, C_{1}$, $C_{2}$ are the solutions of (3.5)-(3.7). In these examples, $C_{2}$ is close to 0 , which explains why $C_{0}=-C_{1}-C_{2}$ is close to $-C_{1}$. The table exhibits $V(8 ; 10)$ and $V(u ; 10)$ with its three components. We note that $V(8 ; 10)$ is a decreasing function of $\sigma$. For the last line $(\sigma=0)$, the formulas (4.7), (4.10) and (4.11) in Avanzi et al (2007) are applied. 
TABLE 1

THE VALUE OF A BARRIER STRATEGY WITH $b=10$. $\left(p(y)=e^{-y}, \lambda=1, c=0.75\right.$ AND $\left.\delta=0.005\right)$

\begin{tabular}{c|c|l}
\hline \hline $\boldsymbol{\sigma}$ & $\boldsymbol{V ( 8 ; 1 0 )}$ & $\boldsymbol{V}(\boldsymbol{u} ; \mathbf{1 0})=C_{\mathbf{0}} e^{\boldsymbol{r}_{0} u}+\boldsymbol{C}_{\mathbf{1}} \boldsymbol{e}^{\boldsymbol{r}_{1} u}+\boldsymbol{C}_{2} \boldsymbol{e}^{\boldsymbol{r}_{2} u}$ \\
\hline 2 & 12.67 & $-18.02808 \cdot e^{-0.10275 u}+18.02808 \cdot e^{0.01867 u}-5 \cdot 10^{-9} \cdot e^{1.46109 u}$ \\
1 & 21.30 & $-22.10986 \cdot e^{-0.20635 u}+22.10986 \cdot e^{0.01803 u}-5 \cdot 10^{-14} \cdot e^{2.68833 u}$ \\
0.5 & 30.76 & $-28.83199 \cdot e^{-0.29793 u}+28.83199 \cdot e^{0.01844 u}-3 \cdot 10^{-34} \cdot e^{7.27948 u}$ \\
0.1 & 36.36 & $-32.98860 \cdot e^{-0.35554 u}+32.98860 \cdot e^{0.01859 u}-3 \cdot 10^{-661} \cdot e^{151.338 u}$ \\
0.005 & 36.63 & $-33.19103 \cdot e^{-0.35858 u}+33.19103 \cdot e^{0.01859 u}-4 \cdot 10^{-260589} \cdot e^{60001 u}$ \\
0 & 36.63 & $-33.19154 \cdot e^{-0.35859 u}+33.19154 \cdot e^{0.01859 u}$ \\
\hline \hline
\end{tabular}

Of course $V(u ; 10)$ for $\sigma=0$ must be the limit of $V(u ; 10)$ if $\sigma \rightarrow 0$. Table 1 illustrates the mechanism of this limit. For $\sigma \rightarrow 0, r_{0}, r_{1}, C_{0}$ and $C_{1}$ converge to the corresponding values in the model without diffusion (last line). In contrast, $C_{2}$ tends to 0 and $r_{2}$ to $\infty$ such that the third component of $V(u ; 10), C_{2} e^{r_{2} u}$, tends to 0 .

\section{THE METHOD OF LAPLACE TRANSFORMS}

The method to calculate $V(u ; b)$ presented in the preceding section is limited to mixtures or (with some adjustments) combinations of exponential gains distributions. In this section we develop a more general method.

To convert the first integral in (2.4) into a convolution integral, we replace the variable $u$ by $z=b-u$, the distance between the dividend barrier and the surplus. The function $W(z ; b)$ is defined as

$$
W(z ; b)=V(b-z ; b), \quad 0 \leq z \leq b .
$$

Note that

$$
W(b ; b)=0
$$

and

$$
W^{\prime}(0 ; b)=-1
$$

by (2.5) and (2.6). The integro-differential equation (2.4) becomes

$$
\begin{aligned}
& \frac{1}{2} \sigma^{2} W^{\prime \prime}(z ; b)+c W^{\prime}(z ; b)-(\lambda+\delta) W(z ; b)+\lambda \int_{0}^{z} W(z-y ; b) p(y) \mathrm{d} y \\
& +\lambda \int_{z}^{\infty}(y-z) p(y) \mathrm{d} y+\lambda W(0 ; b)[1-P(z)]=0 .
\end{aligned}
$$


This equation is valid for $0 \leq z \leq b$. However, the key is to consider this equation for all values $z \geq 0$. The resulting function is denoted by $w(z), z \geq 0$. Let

$$
\hat{f}(\xi)=\int_{0}^{\infty} e^{-\xi x} f(x) \mathrm{d} x
$$

denote the Laplace transform of a function $f(x), x \geq 0$. Taking Laplace transforms in the equation for $w(z)$, we obtain a linear equation for $\widehat{w}(\xi)$. Its solution is

$$
\widehat{w}(\xi)=\frac{\frac{1}{2} \sigma^{2}[-1+\xi w(0)]+c w(0)+\frac{\lambda}{\xi} w(0)[\hat{p}(\xi)-1]-\frac{\lambda}{\xi^{2}}\left[\hat{p}(\xi)-1-\xi \hat{p}^{\prime}(0)\right]}{\frac{1}{2} \sigma^{2}+c \xi-(\lambda+\delta)+\lambda \hat{p}(\xi)} .
$$

This leads us to the following indirect method of calculating $V(u ; b)$. For given $w(0)=V(b ; b)$ we invert $\widehat{w}(\xi)$ to obtain $w(z)$. Then according to (4.2), $b$ is the value of $z$ for which $w(z)=0$, and

$$
V(u ; b)=w(b-u), \quad 0 \leq u \leq b .
$$

Because $V(b ; b)$ is an increasing function of $b, w(0)$ can be any value less than

$$
m=\lim _{b \rightarrow \infty} V(b ; b) .
$$

A closed form expression for $m$ is available. Consider the denominator in (4.5). It is negative for $\xi=0$, tends to infinity for $\xi \rightarrow \infty$, and its second derivative is positive for $\xi>0$. It follows that the denominator in (4.5) has a unique positive zero, which is denoted by $\rho$. Thus

$$
\frac{1}{2} \sigma^{2} \rho^{2}+c \rho-(\lambda+\delta)+\lambda \hat{p}(\rho)=0 .
$$

The function $\widehat{w}(\xi)$ has a pole at $\xi=\rho$ unless the numerator in (4.5) vanishes at $\xi=\rho$. Now consider the case $w(0)=m$. Then $\widehat{w}(\xi)$ must not have a pole, and we conclude that the numerator in (4.5) vanishes at $\xi=\rho$, which leads to

$$
m=\frac{1}{\rho} \frac{\frac{1}{2} \sigma^{2} \rho^{2}+\lambda\left[\hat{p}(\rho)-1-\rho \hat{p}^{\prime}(0)\right]}{\frac{1}{2} \sigma^{2} \rho^{2}+c \rho-\lambda[1-\hat{p}(\rho)]} .
$$

Because of (1.4) and (4.8), the numerator simplifies to $\delta+\rho \mu$, and the denominator simplifies to $\delta$. Thus

$$
m=\frac{\delta+\rho \mu}{\rho \delta}=\frac{1}{\rho}+\frac{\mu}{\delta} .
$$


For $\sigma=0$, an equivalent result was found by Pafumi (1998); see also Avanzi et al (2007).

Remark 4.1. If the gains distribution is of the form (3.1), the zeros of the denominator in (4.5) are $-r_{0},-r_{1}, \ldots,-r_{n+1}$; see (3.3). In particular, $\rho=-r_{0}$.

Remark 4.2. An integro-differential equation like (4.4) appears in the classical model in a different context. As in Gerber and Landry (1998), suppose that the surplus process $(1.1)$ is perturbed by a diffusion. Let $\phi(u)$ denote the expectation of a discounted penalty at ruin, considered a function of the initial surplus $u$. If the penalty consists of the sum of a constant amount $\phi(0)$ and the deficit at ruin, the function $\phi(u)$ satisfies the same integro-differential equation as the function $w(z)$. But the solutions are different. In one case, the equation must be combined with the boundary condition $w^{\prime}(0)=-1$, in the other with the condition that $\phi(u)$ is bounded.

Illustration 4.1. The procedure is illustrated for five different gains distributions, a mixture of two exponential distributions, an exponential distribution, a combination of exponential distributions, the gamma distribution with parameters $\alpha=\beta=2$, and the gamma distribution with parameters $\alpha=\beta=7.3$. The Laplace transforms of $p(y)$ are shown on the left of Table 2 and the following tables. Note that the mean of each distribution is one, while the variance decreases from $5 / 4$ in the first line to $1 / 7.3$ in the fifth line. Throughout, $\lambda=1, c=0.5$ and $\delta=0.002$ such that $\mu=0.5$.

TABLE 2

THE RESUlTiNG VALUE OF $b$ FROM $V(b ; b)=w(0)=100$ $(\lambda=1, c=0.5$ AND $\delta=0.002)$

\begin{tabular}{l|ccccccc}
\hline \hline$\hat{\boldsymbol{p}}(\boldsymbol{\xi})$ & $\boldsymbol{\sigma}=\mathbf{2}^{\mathbf{5}}$ & $\boldsymbol{\sigma}=\mathbf{2}^{\mathbf{2}}$ & $\boldsymbol{\sigma}=\mathbf{2}$ & $\boldsymbol{\sigma}=\mathbf{1}$ & $\boldsymbol{\sigma}=\mathbf{2}^{-\mathbf{2}}$ & $\boldsymbol{\sigma}=\mathbf{2}^{-\mathbf{5}}$ & $\boldsymbol{\sigma}=\mathbf{0}$ \\
\hline$\frac{1}{3} \frac{2}{2+\xi}+\frac{2}{3} \frac{4 / 5}{4 / 5+\xi}$ & 96.576 & 38.166 & 18.829 & 9.939 & 5.139 & 4.635 & 4.626 \\
$\frac{1}{1+\xi}$ & 96.576 & 37.944 & 18.509 & 9.645 & 4.900 & 4.400 & 4.391 \\
$\frac{3}{3 / 2+\xi}-\frac{3}{3+\xi}$ & 96.575 & 37.517 & 17.848 & 8.988 & 4.327 & 3.829 & 3.821 \\
$\left(\frac{2}{2+\xi}\right)^{2}$ & 96.575 & 37.463 & 17.768 & 8.915 & 4.275 & 3.780 & 3.771 \\
$\left(\frac{7.3}{7.3+\xi}\right)^{7.3}$ & 96.573 & 37.091 & 17.165 & 8.316 & 3.810 & 3.330 & 3.322 \\
\hline \hline
\end{tabular}

We choose $w(0)=100$. This value is admissible, because $100<250=\frac{\mu}{\delta}<m$ by (4.10). Table 2 shows $b$, the zero of $w(z)$. It appears that $b$ increases with $\sigma$ and with the variance of the gains distribution. 
With Maple or Mathematica, the inversion of $\widehat{w}(\xi)$ works well if $\hat{p}(\xi)$ is a rational function. For the fifth example, $\hat{p}(\xi)$ is not a rational function. To facilitate the inversion, $\hat{p}(\xi)$ was replaced by

$$
\left(\frac{1}{1+v \xi}\right)^{7}\left(\frac{1}{1+(1-7 v) \xi}\right)
$$

which is a rational function of $\xi$. Here $v$ is determined by the requirement that the variance of the approximating distribution is $1 / 7.3$, the variance of the original distribution. This is the requirement that

$$
7 v^{2}+(1-7 v)^{2}=\frac{1}{7.3} .
$$

Hence, $v=1 / 7.172$. This method is based on interpolation between successive Erlang distributions; see Avanzi et al (2007).

\section{THE OPTIMAL DIVIDEND BARRIER}

We denote by $b^{*}$ the optimal value of $b$, that is the value of $b$ which maximizes $V(u ; b)$ for given value of $u$. Thus

$$
\left.\frac{\partial V(u ; b)}{\partial b}\right|_{b=b^{*}}=0 .
$$

We shall show how $b^{*}$ and $V\left(u, b^{*}\right)$ can be calculated.

If we differentiate the identity (2.6), we obtain another identity:

$$
V^{\prime \prime}(b-; b)+\left.\frac{\partial^{2} V(u ; b)}{\partial u \partial b}\right|_{u=b-}=0 .
$$

For $b=b^{*}$, the second term vanishes because of (5.1). It follows that

$$
V^{\prime \prime}\left(b^{*}-; b^{*}\right)=0 .
$$

The interpretation is as follows. From (2.6) and (2.3) we see that the function $V(u ; b)$ is continuously differentiable at the junction $u=b$. Now (5.3) shows that $V\left(u, b^{*}\right)$ is twice continuously differentiable at $u=b^{*}$. Such a result is known as a high contact or smooth pasting condition for optimality.

Because $V(b ; b)$ increases from 0 to $m$ as $b$ goes from 0 to $\infty$, it follows from (2.11) and (4.10) that $\frac{1}{2} \sigma^{2} V^{\prime \prime}(b-; b)$ increases from $-\mu$ to $\delta / \rho$ as $b$ goes from 0 to $\infty$. Thus $b^{*}$ can be characterized as the unique and positive zero of the function $V^{\prime \prime}(b-; b)$. 
From (5.3) and (2.11) with $b=b^{*}$, we conclude that

$$
V\left(b^{*} ; b^{*}\right)=\frac{\mu}{\delta} \text {. }
$$

For the pure diffusion model, such a result has been obtained by Gerber (1972). For model (1.2) it has been found by Avanzi et al (2007).

Formula (5.4) is crucial for implementing the method of Laplace transforms described in Section 4. Formula (5.4) is equivalent to $W\left(0 ; b^{*}\right)=\mu / \delta$. Thus we procede as follows. In (4.5) we set $w(0)=\mu / \delta$ and obtain the function $w(z)$ by inversion of its Laplace transform. Then $b^{*}$ is the zero of $w(z)$, and

$$
V\left(u ; b^{*}\right)=w\left(b^{*}-u\right), \quad 0 \leq z \leq b^{*} .
$$

\section{TABLE 3}

THE OPTIMAL DIVIDEND BARRIER $b^{*}$

$$
(\lambda=1, c=0.5 \text { AND } \delta=0.002)
$$

\begin{tabular}{l|ccccccc}
\hline \hline$\hat{\boldsymbol{p}}(\boldsymbol{\xi})$ & $\boldsymbol{\sigma}=\mathbf{2}^{\mathbf{5}}$ & $\boldsymbol{\sigma}=\mathbf{2}^{\mathbf{2}}$ & $\boldsymbol{\sigma}=\mathbf{2}$ & $\boldsymbol{\sigma}=\mathbf{1}$ & $\boldsymbol{\sigma}=\mathbf{2}^{-\mathbf{2}}$ & $\boldsymbol{\sigma}=\mathbf{2}^{-\mathbf{5}}$ & $\boldsymbol{\sigma}=\mathbf{0}$ \\
\hline$\frac{1}{3} \frac{2}{2+\xi}+\frac{2}{3} \frac{4 / 5}{4 / 5+\xi}$ & 240.320 & 87.772 & 42.283 & 22.351 & 11.948 & 10.879 & 10.861 \\
$\frac{1}{1+\xi}$ & 240.317 & 87.203 & 41.476 & 21.597 & 11.327 & 10.269 & 10.251 \\
$\frac{3}{3 / 2+\xi}-\frac{3}{3+\xi}$ & 240.313 & 86.126 & 39.849 & 19.972 & 9.891 & 8.841 & 8.823 \\
$\left(\frac{2}{2+\xi}\right)^{2}$ & 240.313 & 85.990 & 39.649 & 19.788 & 9.756 & 8.871 & 8.694 \\
$\left(\frac{7.3}{7.3+\xi}\right)^{7.3}$ & 240.310 & 85.062 & 38.188 & 18.323 & 8.584 & 7.577 & 7.560 \\
\hline \hline
\end{tabular}

TABLE 4

THE MAXIMAL EXPECTED DISCOUNTED DIVIDENDS $V\left(2 ; b^{*}\right)$ $(\lambda=1, c=0.5$ AND $\delta=0.002)$

\begin{tabular}{l|ccccccc}
\hline \hline$\hat{p}(\xi)$ & $\boldsymbol{\sigma}=\mathbf{2}^{\mathbf{5}}$ & $\boldsymbol{\sigma}=\mathbf{2}^{\mathbf{2}}$ & $\boldsymbol{\sigma}=\mathbf{2}$ & $\boldsymbol{\sigma}=\mathbf{1}$ & $\boldsymbol{\sigma}=\mathbf{2}^{\mathbf{2}}$ & $\boldsymbol{\sigma}=\mathbf{2}^{\mathbf{- 5}}$ & $\boldsymbol{\sigma}=\mathbf{0}$ \\
\hline$\frac{1}{3} \frac{2}{2+\xi}+\frac{2}{3} \frac{4 / 5}{4 / 5+\xi}$ & 2.2 & 21.5 & 64.1 & 127.8 & 195.9 & 204.3 & 204.5 \\
$\frac{1}{1+\xi}$ & 2.2 & 21.7 & 65.8 & 132.1 & 201.5 & 209.8 & 210.0 \\
$\frac{3}{3 / 2+\xi}-\frac{3}{3+\xi}$ & 2.2 & 22.2 & 69.4 & 141.9 & 214.2 & 222.1 & 222.3 \\
$\left(\frac{2}{2+\xi}\right)^{2}$ & 2.2 & 22.3 & 69.8 & 143.1 & 215.4 & 223.2 & 223.4 \\
$\left(\frac{7.3}{7.3+\xi}\right)^{7.3}$ & 2.2 & 22.7 & 73.3 & 152.8 & 225.2 & 232.0 & 232.2 \\
\hline \hline
\end{tabular}


Illustration 5.1. We continue the numerical examples of Section 4. To obtain the optimal dividend barrier $b^{*}$, it suffices to set $w(0)=\mu / \delta=250$. Then $b^{*}$ is the zero of $w(z)$. Table 3 shows the optimal dividend barrier $b^{*}$, and Table 4 shows $V\left(2 ; b^{*}\right)$, the maximal expected value of the discounted dividends, if the initial surplus is 2 .

Remark 5.1. In Section 3 it was assumed that the gains distribution is a mixture of exponential distributions. In this case, the optimal dividend barrier $b^{*}$ can be obtained more directly as a solution of an implicit equation. From (5.1) and (3.2) it follows that

$$
C_{k}^{\prime}\left(b^{*}\right)=0, \quad k=0,1, \ldots, n+1 .
$$

Now we differentiate (3.5) and (3.7) with respect to $b$, set $b=b^{*}$ and use (5.6) to see that

$$
\sum_{k=0}^{n+1} C_{k}\left(b^{*}\right) \frac{r_{k}^{2}}{\beta_{i}-r_{k}} e^{r_{k} b^{*}}=0, \quad i=1, \ldots, n,
$$

and

$$
\sum_{k=0}^{n+1} C_{k}\left(b^{*}\right) r_{k}^{2} e^{r_{k} b^{*}}=0 .
$$

Together with (3.6), (5.7) and (5.8) constitute a homogeneous system of $n+2$ linear equations for $C_{0}\left(b^{*}\right), C_{1}\left(b^{*}\right), \ldots, C_{n+1}\left(b^{*}\right)$. It follows that the determinant of the coefficient matrix of this system must vanish. For example, for $n=1$, this is the condition that

$$
\operatorname{det}\left[\begin{array}{ccc}
\frac{r_{0}^{2}}{\beta-r_{0}} e^{r_{0} b^{*}} & \frac{r_{1}^{2}}{\beta-r_{1}} e^{r_{1} b^{*}} & \frac{r_{2}^{2}}{\beta-r_{2}} e^{r_{2} b^{*}} \\
r_{0}^{2} e^{r_{0} b^{*}} & r_{1}^{2} e^{r_{1} b^{*}} & r_{2}^{2} e^{r_{2} b^{*}} \\
1 & 1 & 1
\end{array}\right]=0 .
$$

Solving the determinant equation is an alternative to solving the equation $w\left(b^{*}\right)=0$ after inversion of $\widehat{w}(\xi)$ with $w(0)=\mu / \delta$.

\section{RESCALING WITH COMPENSATION}

In this section we consider a family of models. The distribution of an individual gain is rescaled, and the Poisson parameter is adjusted such that the expected gain per unit time is unchanged. 
Without loss of generality, we assume that the original probability density function $p(x)$ has mean one. For $\varphi>0$, we introduce

$$
p_{\varphi}(x)=\varphi p(\varphi x), \quad x>0,
$$

which has mean $1 / \varphi$. Note that

$$
\hat{p}_{\varphi}(\xi)=\hat{p}(\xi / \varphi)
$$

The Poisson parameter is set to be $\varphi$, so that the expected gain per unit time is one, independently of $\varphi$. Furthermore, $0<c<1$ and $\mu=1-c$ do not depend on $\varphi$.

We consider two limits. For $\varphi \rightarrow \infty, S(t) \rightarrow t$ and hence the process (1.3) becomes the Wiener process with drift $\mu$ and variance per unit time $\sigma^{2}$. For this case, explicit results are available, in particular

$$
V(u ; b)=\frac{e^{r_{1} u}-e^{r_{0} u}}{r_{1} e^{r_{1} b}-r_{0} e^{r_{0} b}}
$$

and

$$
b^{*}=\frac{1}{r_{1}-r_{0}} \ln \frac{r_{0}^{2}}{r_{1}^{2}}
$$

where $-r_{0}$ and $-r_{1}$ are the zeros of the denominator in (4.5) with $\lambda=0$; see, for example, Gerber and Shiu (2004).

Now we consider the limit $\varphi \rightarrow 0$. It is clear that the probabilistic model (1.3) does not make sense in the limit. Nevertheless, quantities of interest such as $b^{*}$ and $V\left(u ; b^{*}\right)$ have limiting values, which could be used as bounds or estimators in extreme situations where the gains are big but rare. To determine these values, we start with the limit of the Laplace transform of $w(z)$. From (4.5) we see that the limit for $\varphi \rightarrow 0$ is

$$
\widehat{w}(\xi)=\frac{\frac{1}{2} \sigma^{2}[-1+\xi w(0)]+c w(0)-\frac{1}{\xi}}{\frac{1}{2} \sigma^{2} \xi^{2}+c \xi-\delta} .
$$

For $w(0)=\mu / \delta$, this can be written as

$$
\begin{aligned}
\widehat{w}(\xi) & =\frac{\mu}{\delta} \cdot \frac{1}{\xi}-\frac{\frac{1}{2} \sigma^{2}+\frac{c}{\xi}}{\frac{1}{2} \sigma^{2} \xi^{2}+c \xi-\delta} \\
& =\frac{\mu}{\delta} \cdot \frac{1}{\xi}-\frac{\xi+\alpha}{\xi\left(\xi+r_{0}\right)\left(\xi+r_{2}\right)},
\end{aligned}
$$


where $\alpha=\frac{2 c}{\sigma^{2}}$ and $-r_{0},-r_{2}$ are the zeros of the denominator in (6.3). Applying the method of partial fractions, we obtain

$$
\widehat{w}(\xi)=\frac{\mu}{\delta} \cdot \frac{1}{\xi}-\frac{C_{0}}{\xi+r_{0}}-\frac{C_{1}}{\xi}-\frac{C_{2}}{\xi+r_{2}}
$$

with

$$
C_{0}=\frac{r_{0}-\alpha}{r_{0}\left(r_{2}-r_{0}\right)}, \quad C_{1}=\frac{\alpha}{r_{0} r_{2}}, \quad C_{0}=\frac{r_{2}-\alpha}{r_{2}\left(r_{0}-r_{2}\right)} .
$$

Note that $C_{0}+C_{1}+C_{2}=0$. From (6.5) it follows that

$$
w(z)=\frac{\mu}{\delta}-\left(C_{0} e^{-r_{0} z}+C_{1}+C_{2} e^{-r_{2} z}\right) .
$$

Now the limiting value of $b^{*}$ is determined from the condition that $w\left(b^{*}\right)=0$, and $V\left(u ; b^{*}\right)=w\left(b^{*}-u\right)$.

Illustration 6.1. Assume that $p(x)=e^{-x}$, that is $p_{\varphi}(x)=\varphi e^{-\varphi x}$, and suppose that $c=0.75, \sigma=0.5$, and $\delta=0.005$. Thus $\mu=0.25$ and $V\left(b^{*} ; b^{*}\right)=50$. Table 5 shows $b^{*}, V\left(4 ; b^{*}\right)$, and also $r_{0}, r_{1}, r_{2}$ as functions of $\varphi$. For $0<\varphi<\infty$, the calculations are based on the formulas of Section 3 with $n=1$. The first and last lines

TABLE 5

$b^{*}$ AND $V\left(u ; b^{*}\right)$ AS FUNCTIONS OF $\varphi$

$(\lambda=1, c=0.75, \sigma=0.5$ AND $\delta=0.005)$

\begin{tabular}{c|c|c|c|c|c}
\hline \hline $\boldsymbol{\rho}$ & $\boldsymbol{b}^{*}$ & $-\boldsymbol{r}_{\mathbf{0}}$ & $\boldsymbol{r}_{\mathbf{1}}$ & $\boldsymbol{r}_{\mathbf{2}}$ & $\boldsymbol{V ( 4 ; \boldsymbol { b } ^ { * } )}$ \\
\hline 0 & 43.20 & 0.006659 & 0 & 6.006659 & 5.257 \\
0.001 & 43.10 & 0.007839 & 0.000849 & 6.007990 & 5.289 \\
0.1 & 35.43 & 0.051613 & 0.012624 & 6.138989 & 8.492 \\
0.5 & 22.55 & 0.173157 & 0.017316 & 6.655803 & 19.591 \\
1 & 16.84 & 0.297928 & 0.018444 & 7.279485 & 28.464 \\
10 & 6.76 & 1.185714 & 0.019652 & 17.16606 & 46.988 \\
100 & 4.80 & 1.874168 & 0.019789 & 107.8544 & 49.190 \\
1000 & 4.56 & 2.003961 & 0.019802 & 1007.984 & 49.436 \\
$\infty$ & 4.54 & 2.019804 & 0.019804 & & 49.464 \\
\hline \hline
\end{tabular}

exhibit the limiting values discussed in this section. We note that $b^{*}$ is decreasing in $\varphi$ and that $V\left(4 ; b^{*}\right)$ is increasing in $\varphi$. For large values of $\varphi, b^{*}$ is close to 4 . This explains why $V\left(4 ; b^{*}\right)$ is close to $V\left(b^{*} ; b^{*}\right)=50$. 


\title{
ACKNOWLEDGEMENTS
}

The authors are grateful to two anonymous referees, as well as to Hansjörg Albrecher, François Dufresne, Marc Goovaerts and Elias Shiu. Their comments contributed to substantial improvements of the paper.

\section{REFERENCES}

AvAnZI, B. (2008) A review of modern collective risk theory with dividend strategies. UNSW Australian School of Business Research Paper No. 2008ACTL09.

Avanzi, B., Gerber, H.U. and Shiu, E.S.W. (2007) Optimal dividends in the dual model. Insurance: Mathematics and Economics 41(1), 111-123.

Azcue, P. and Muler, N. (2005) Optimal reinsurance and dividend distribution policies in the Cramér-Lundberg model. Mathematical Finance 15(2), 261-308.

Bayraktar, E. and Egami, M. (2008) Optimizing venture capital investments in a jump diffusion model. Mathematical Methods of Operations Research 67(1), 21-42.

Bühlmann, H. (1970) Mathematical Methods in Risk Theory. Springer-Verlag, Berlin Heidelberg New York.

DE FinetTI, B. (1957) Su un'impostazione alternativa della teoria collettiva del rischio. Transactions of the XVth International Congress of Actuaries 2, 433-443.

GERBER, H.U. (1972) Games of economic survival with discrete- and continuous-income processes. Operations Research 20(1), 37-45.

Gerber, H.U. and LANDRY, B. (1998) On the discounted penalty at ruin in a jump-diffusion and the perpetual put option. Insurance: Mathematics and Economics 22(3), 263-276.

Gerber, H.U. and Shiu, E.S.W. (2004) Optimal dividends: Analysis with Brownian motion. North American Actuarial Journal 8(1), 1-20.

MiYasawa, K. (1962) An economic survival game. Journal of the Operations Research Society of Japan 4(3), 95-113.

PAfumi, G. (1998) Discussion on H.U. Gerber and E.S.W. Shiu's "On the time value of ruin". North American Actuarial Journal 2(1), 5-76.

Schmidui, H. (2008) Stochastic Control in Insurance. Probability and its Applications. SpringerVerlag, London.

\author{
Benjamin Avanzi (Corresponding Author) \\ Actuarial Studies \\ Australian School of Business \\ UNSW Sydney NSW 2052 \\ Australia \\ Email:b.avanzi@unsw.edu.au
}

HANS U. Gerber

Distinguished Visiting Professor at The University of Hong Kong

University of Lausanne, Faculty of Business and Economics

1015 Lausanne

Switzerland

Email:hgerber@unil.ch 